\title{
Внутрицентровая релаксация мелких доноров мышьяка В деформированном германии. Инверсия населенностей при оптическом возбуждении
}

\author{
(C) В.В. Цыпленков, В.Н. Шастин
}

Институт фризики микроструктур Российской академии наук, 603950 Нижний Новгород, Россия

E-mail: Tsyplenkov1@yandex.ru

(Получена 25 апреля 2018 г. Принята к печати 7 мая 2018 г.)

\begin{abstract}
Проводится расчет скоростей релаксации нижних возбужденных состояний $1 s(T), 2 p_{0}, 2 s, 3 p_{0}, 2 p_{ \pm}$ доноров мышьяка в кристалле германия при взаимодействии с длинноволновыми акустическими фононами в зависимости от одноосной деформации сжатия в кристаллографическом направлении [111]. На основе вычисленных времен произведены оценки населенностей состояний при оптическом возбуждении. Теоретически показано, что оптическое возбуждение среды формирует инверсную населенность уровней донора мышьяка и при нулевом значении деформации приводит к возможности реализации четырехуровневой лазерной схемы с излучательным переходом между состояниями $2 p$ и триплетным состоянием $1 s$. В условиях оптического возбуждения излучением $\mathrm{CO}_{2}$-лазера оценочное значение ожидаемого коэффициента усиления в среде при концентрации доноров $2 \cdot 10^{15} \mathrm{~cm}^{-3}$ составляет $\sim 0.35 \mathrm{~cm}^{-1}$ на частоте 1.98 ТГц в случае, если рабочим переходом является $2 p_{ \pm} \rightarrow 1 s(T)$, и 1.25 ТГц, если рабочий переход $2 p_{0} \rightarrow 1 s(T)$.
\end{abstract}

DOI: 10.21883/FTP.2018.12.46759.39

\section{1. Введение}

В настоящее время активность многих исследовательских групп направлена на исследование кулоновских центров в полупроводниках. Основное внимание, как правило, уделяется поиску квантовых когерентных состояний на основе примесных центров и методов управления ими (см., например, [1-6]), что обусловлено возможностью создания „одноатомных“ транзисторов и элементов новых квантовых устройств. В этом контексте проведенные в настоящей работе теоретические исследования релаксационных процессов в легированном мелкими донорами мышьяка кристалле германия, в том числе в условиях одноосной деформации сжатия в кристаллографическом направлении $\{111\}$, показывающие возможность создания нового источника терагерцового излучения на внутрицентровых переходах, становятся очень актуальными. С точки зрения интегрирования появляющихся новых устройств и расширения их возможностей очень важно иметь лазер, а для класса „одноатомных“ устройств — лазер на внутрицентровых переходах, излучение которого могло бы резонансно влиять на свойства такого объекта. Таким образом, в случае необходимости проведения измерения состояния донорного электрона в германии необходимо использовать квант излучения, соответствующий переходам между локализованными состояниями донора или его ионизации. В первом случае самым подходящим кандидатом на роль источника такого зондирующего сигнала можно предложить лазер на внутрицентровых переходах германия ввиду резонансности, а следовательно, и эффективности взаимодействия. Кроме того, использование доноров в объемном германии как основы активной среды позволяет иметь стабильный источник ввиду независимости частоты перехода от технологического процесса изготовления активной среды.

Лазеры на основе объемного германия имеют и самостоятельный интерес. В группе Института физики микроструктур РАН уже на протяжении ряда лет разрабатываются альтернативные источники терагерцового (ТГц) излучения, в которых рабочими являются внутрицентровые переходы мелких примесных центров в кремнии. К настоящему времени реализовано стимулированное излучение доноров V группы (сурьма, фосфор, мышьяк, висмут) и акцепторов бора в объемном кремнии при оптическом возбуждении и, в частности, при фотоионизации этих центров излучением TEA $\mathrm{CO}_{2}$-лазера [7,8]. Изучены оптимальные условия генерации в условиях одноосной деформации, достигнуто существенное увеличение коэффициента усиления и снижения внутренних потерь в образцах [9]. По сравнению с квантово-каскадными лазерами такие источники просты в изготовлении и обслуживании, имеют большую выходную мощность, диапазон частот 4.5-6.0 ТГц, и ввиду того, что генерация развивается на переходах между уровнями доноров, частоты источников заведомо являются резонансными для „одноатомных“ транзисторов на основе доноров (см., например, [10]), а значит, наиболее подходят для управления свойствами таких приборов. Недостатком источников является отсутствие на данный момент непрерывного режима генерации, так как мощность непрерывного фотовозбуждения, необходимая для поддержания режима генерации, приводит к перегреву образца.

Проведенные в настоящей работе теоретические оценки говорят о возможности создания подобных источников на основе объемного германия, легированного 
мелкими донорами мышьяка. Состояния доноров в германии имеют значительно большее время жизни [11] по сравнению с кремнием, и это позволяет надеяться, что в случае обнаружения лазерного эффекта в германии на внутрицентровых переходах можно реализовать непрерывный режим генерации, так как пороговое значение мощности возбуждения должно быть значительно ниже, чем в кремниевых лазерах.

\section{2. Теоретическая модель}

\section{1. Волновые функции состояний доноров}

Описание волновых функций состояний доноров в германии осуществлялось в рамках приближения эффективных масс. Зона проводимости кристалла германия имеет четыре эквивалентных минимума (долины) в $k$-пространстве, вследствие чего состояния кулоновского центра в германии четырехкратно вырождены (см., например, [12]). Однако, как известно, потенциал примесного центра отличается от кулоновского, он помимо кулоновской составляющей имеет дополнительную часть - короткодействующий потенциал, или потенциал центральной ячейки, который быстро спадает с увеличением расстояния от центра. Наличие такого потенциала приводит к снятию вырождения и расщеплению состояний доноров (химический сдвиг). Так как действие короткодействующего потенциала сильно локализовано, как правило, не выходит за рамки одной центральной ячейки, то энергетический сдвиг существен только для состояний с волновой функцией, не равной нулю при $\mathbf{r}=0$. Это позволяет не учитывать влияние короткодействующего потенциала для всех рассматриваемых в данной работе состояний кроме состояния $1 s$ и считать их четырехкратно вырожденными. Конечно, состояние $2 s$ мелких доноров в германии также подвергается расщеплению под действием потенциала центральной ячейки, но это расщепление невелико и не влияет существенно на схему релаксации возбужденных доноров; поэтому в настоящей работе этим расщеплением пренебрегается и состояние $2 s$ также считается четырехкратно вырожденным. Состояние же $1 s$ в недеформированном кристалле расщепляется на синглет, образованный равными вкладами всех долин зоны проводимости германия, и триплет, состояния которого образованы не равными вкладами различных долин (см., например, [12]).

Волновая функция донорного центра в германии в рамках метода эффективных масс может быть записана в виде

$$
\Psi(\mathbf{r})=\sum_{i=1}^{4} \alpha_{i} F(\mathbf{r}) \varphi_{i}(\mathbf{k}, \mathbf{r}),
$$

где суммирование проводится по всем четырем долинам зоны проводимости германия, коэффициенты $\alpha_{i}$ определяют весовой вклад $i$-й долины в состояние донора, $F(\mathbf{r})$ - огибающая волновой функции, определяемая гамильтонианом, в который не входит периодический

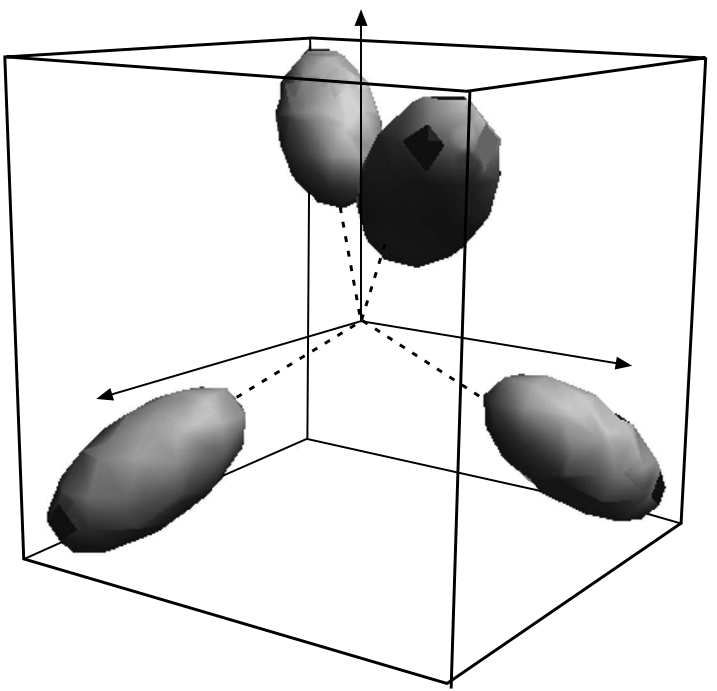

Рис. 1. Схема долин зоны проводимости кристалла германия.

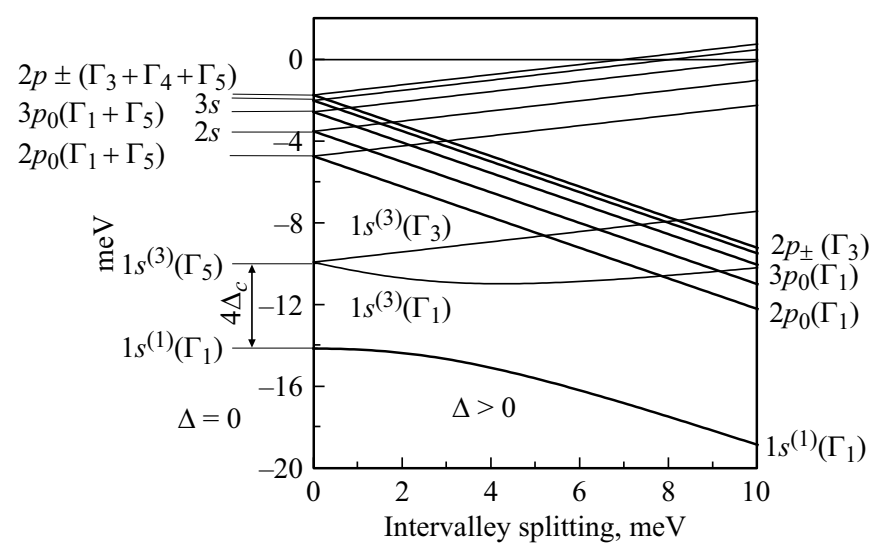

Рис. 2. Схема уровней донора мышьяка в германии при деформации $(\Delta>0)$ в кристаллографическом направлении [111] и в недеформированном кристалле $(\Delta=0)$. Деформация кристалла выражена в мэВ (междолинное расщепление). Давление в 1 кбар соответствует междолинному расщеплению $\sim 10.81 \mathrm{мэB}$.

потенциал решетки кристалла, а вместо массы электрона входят его эффективные массы, $\varphi_{i}(\mathbf{k}, \mathbf{r})$ - волновая функция Блоха на дне $i$-й долины.

На рис. 1 приведена схема долин зоны проводимости германия. Долины имеют цилиндрическую симметрию и ориентированы вдоль кристаллографических направлений $[111],[\overline{1} 1 \overline{1}],[1 \overline{1} \overline{1}]$ и $[\overline{1} \overline{1} 1]$. Уровни энергии донора мышьяка в германии в зависимости от междолинного расщепления представлены на рис. 2.

Распределение состояний мелких доноров по долинам зоны проводимости германия (коэффициенты $\alpha_{i}$ ) в зависимости от междолинного расщепления $(\Delta)$ в направлении [111] дается выражениями в табл. 1 [12].

В качестве огибающих волновых функций состояний были использованы пробные волновые функции $[13,14]$ 
Таблица 1. Зависимость коэффициентов $\alpha_{i}$, определяющих долинные вклады в волновую функцию состояния донора, от междолинного расщепления

\begin{tabular}{c|c}
\hline $1 s^{(1)}\left(\Gamma_{1}\right)$ & $\alpha_{1}=\frac{1}{\sqrt{2}} a^{+} ; \alpha_{2}=\alpha_{3}=\alpha_{4}=a^{-}$ \\
\hline $1 s^{(3)}\left(\Gamma_{3}\right)$ & $\alpha_{1}=0 ; \alpha_{2}=\frac{1}{\sqrt{2}} ; \alpha_{3}=-\frac{1}{\sqrt{2}} ; \alpha_{4}=0$ \\
& $\alpha_{1}=0 ; \alpha_{2}=\frac{1}{\sqrt{2}} ; \alpha_{3}=-\frac{1}{\sqrt{2}} ; \alpha_{4}=0$ \\
\hline $1 s^{(3)}\left(\Gamma_{1}\right)$ & $\alpha_{1}=\frac{1}{\sqrt{2}} a^{-} ; \alpha_{2}=\alpha_{3}=\alpha_{4}=a^{+}$ \\
\hline \multicolumn{3}{c}{$a^{ \pm}=\sqrt{1 \pm \frac{x-1 / 2}{\sqrt{1-x+x^{2}}}}, x=\frac{\Delta}{\Delta_{c}}$}
\end{tabular}

Примечание. $\Delta_{c}-$ химический сдвиг, определяемый как четверть энергетического зазора между основным состоянием и триплетным состоянием $1 s$

Таблица 2. Значения параметров $a$ и $b$ (в единицах постоянной решетки $a_{0}$ ), определяющих локализацию волновых функций донора в пространстве

\begin{tabular}{c|c|c}
\hline Состояние & $a$ & $b$ \\
\hline $2 p_{0}$ & 14.26 & 5.45 \\
$2 s$ & 22.87 & 8.07 \\
$3 p_{0}$ & 21.4 & 8.18 \\
$2 p_{ \pm}$ & 28.24 & 11.43
\end{tabular}

с варьируемыми параметрами $a$ и $b$, определяющими локализацию состояния в пространстве. Далее эти функции представлены в пространстве волновых векторов в системе координат, в которой долина ориентирована вдоль оси $x$ (использование $k$-представления более удобно для вычисления вероятностей переходов при взаимодействии с фононами):

$$
\begin{gathered}
F_{1 s}=\frac{2 a \sqrt{2 b}}{\pi} \frac{1}{\left[\left(k_{x} b\right)^{2}+\left(k_{y} a\right)^{2}+\left(k_{z} a\right)^{2}+1\right]^{2}}, \\
F_{2 p_{0}}=\frac{8 \sqrt{2} a b^{3 / 2}}{\pi} \frac{k_{x}}{\left[\left(k_{x} b\right)^{2}+\left(k_{y} a\right)^{2}+\left(k_{z} a\right)^{2}+1\right]^{3}}, \\
F_{2 s}=\frac{4 \sqrt{2 a b^{2}}}{\pi}\left\{\frac{1}{\left[\left(k_{x} b\right)^{2}+\left(k_{y} a\right)^{2}+\left(k_{z} a\right)^{2}+1\right]^{2}}\right. \\
F_{3 p_{0}}=\frac{32 a b^{3 / 2}}{\sqrt{3} \pi} k_{x} \frac{2\left[\left(k_{x} b\right)^{2}+\left(k_{y} a\right)^{2}+\left(k_{z} a\right)^{2}\right]-1}{\left[\left(k_{x} b\right)^{2}+\left(k_{y} a\right)^{2}+\left(k_{z} a\right)^{2}+1\right]^{4}}, \\
F_{2 p_{ \pm}}=\frac{8 \sqrt{2} a^{2} \sqrt{b}}{\pi} \frac{k_{z}+i k_{y}}{\left[\left(k_{x} b\right)^{2}+\left(k_{y} a\right)^{2}+\left(k_{z} a\right)^{2}+1\right]^{3}} .
\end{gathered}
$$

Значения параметров $a$ и $b$, вычисленные методом минимизации энергии состояния с гамильтонианом эффективных масс, известны в литературе. Вычисленные таким образом значения в данной работе использованы для возбужденных состояний $2 p_{0}, 2 s, 3 p_{0}, 2 p_{ \pm}$. Значения параметров $a$ и $b$ для этих состояний, использованных в данной работе, представлены в табл. 2 [14].

Состояние $1 s$, как сказано выше, подвержено существенному химическому сдвигу, и такой подход определения параметров $a$ и $b$ был бы не верен, так как гамильтониан эффективных масс не учитывает короткодействующий потенциал центральной ячейки. В связи с тем, что вид этого потенциала не известен, в данной работе использован квазиклассический подход определения $a$ и $b$ [15], основанный на сравнении асимптотического поведения волновой функции на больших расстояниях от примесного центра со спаданием функций $1 s$-состояний, определяемой выражением из (2). Вычисление этим методом происходит с опорой на фактическое значение энергии состояния, которое известно. Такой подход дает следующие выражения для $a$ и $b$ :

$$
a=\sqrt{\frac{\hbar^{2}}{2 m_{t} E}} ; \quad b=\sqrt{\frac{\hbar^{2}}{2 m_{l} E}} .
$$

Важно отметить, что энергия $1 s$-состояний изменяется относительно дна долин при деформации кристалла, поэтому параметры $a$ и $b$ также зависят от прикладываемого давления, что учитывается в данной работе.

\section{2. Переходы между состояниями доноров В германии с излучением фононов}

Электрон-фононное взаимодействие в кристалле германия может приводить как к внутридолинным, так и к междолинным переходам между электронными состояниями. Так как энергия основного состояния мелких доноров V группы в германии не превышает 15 мэВ, междолинные внутрицентровые переходы принципиально возможны лишь при взаимодействии с ТА-фононами (поперечными акустическими), волновой вектор которых лежит в окрестности точки $X$ зоны Бриллюэна и которые имеют энергию 10 мэВ. Групповая скорость ТА-фононов, излучаемых при междолинных переходах в германии, мала, поэтому междолинные переходы существенны лишь в очень узких диапазонах энергий ( 0.5 мэВ). В донорах мышьяка в недеформированном кристалле германия с такой точностью резонансные условия могут реализоваться только на переходах из узкого энергетического пояска высоковозбужденных состояний, находящихся в долях мэВ от дна зоны проводимости, либо из самого дна зоны проводимости в $1 s$-состояния триплетной группы. В настоящей работе предполагается, что релаксация таких высоковозбужденных состояний определяется главным образом внутридолинными переходами при взаимодействии с длинноволновыми акустическими фононами и междолинные процессы электрон-фононного взаимодействия не учитываются. На зависимостях же темпов переходов от деформации междолинные переходы должны проявляться в виде узких пиков с шириной $~ 50$ бар. Согласно проведенным 
авторами предварительным оценкам, максимальные значения темпов междолинных переходов из всех состояний типа $2 p$ приблизительно на порядок меньше, чем темпы внутридолинных переходов из тех же состояний. Темп междолинного перехода из состояния $2 s$ в основное состояние донора может превосходить скорости внутридолинных процессов релаксации. Этот междолинный процесс релаксации может быть эффективен в диапазоне давлений от $\sim 80$ до $\sim 150$ бар. Максимум междолинного темпа перехода $2 s-1 s^{(1)}\left(\Gamma_{1}\right)$ достигается при междолинном расщеплении $\sim 1.5$ мэВ. В данной работе не проводится детальный анализ междолинных процессов электрон-фононного взаимодействия.

Вычисление темпов излучения акустических фононов на внутрицентровых переходах осуществлялось в рамках обычного подхода с использованием золотого правила Ферми. Закон дисперсии длинноволновых акустических фононов, участвующих во внутридолинных переходах, считается изотропным. Принимается, что оператор электрон-фононного взаимодействия имеет вид

$$
H_{\text {int }}=\sum_{\mathbf{a} \alpha} \mathbf{F}\left(\mathbf{r}-\mathbf{R}_{\mathbf{a} \alpha}\right) \mathbf{Q}_{\mathbf{a} \alpha},
$$

где $\mathbf{F}\left(\mathbf{r}-\mathbf{R}_{\mathbf{a} \alpha}\right)$ имеет смысл силы, действующей со стороны иона решетки, положение которого $\mathbf{R}_{\mathbf{a} \alpha}$, на электрон с координатами $\mathbf{r}, \alpha-$ номер этого иона в ячейке, положение которой в кристалле задается вектором а, $\mathbf{Q}_{\mathbf{a} \alpha}$ - вектор смещения иона кристаллической решетки. Суммирование ведется по всем атомам кристаллической решетки. Используя известные соотношения квантовой механики, вектор $\mathbf{Q}_{\mathbf{a} \alpha}$ можно представить в виде

$$
\mathbf{Q}_{\mathbf{a} \alpha}=\sum_{s \mathbf{q}} \sqrt{\frac{\hbar}{2 N V \rho \omega_{\mathbf{q}}}} \mathbf{e}_{s \mathbf{q}} a_{\mathbf{q} s}^{+} e^{-i \mathbf{q} \mathbf{a}}+C . C .
$$

Эта формула представляет собой разложение смещения атома кристаллической решетки по фононам, где $s-$ номер фононной ветви, q - волновой вектор фонона, $\mathbf{e}_{s \mathbf{q}}-$ его поляризация, $a_{\mathbf{q} s}^{+}-$оператор рождения фонона с волновым вектором q и принадлежащим ветви с номером $s, N$ - число элементарных ячеек в кристалле, $V$ - объем кристалла, $\rho$ - плотность кристалла.

Матричный элемент перехода между состояниями донора с номерами $m$ и $n$ с излучением фонона с волновым вектором q имеет вид

$$
\begin{aligned}
M_{n m} & =\int\left\langle n_{q}+1\right| \Psi_{n}^{*} \\
& \times\left(\sum_{\mathbf{a} \alpha} \mathbf{F}\left(\mathbf{r}-\mathbf{R}_{\mathbf{a} \alpha}\right) \mathbf{e}_{s \mathbf{q}} \sqrt{\frac{\hbar}{2 N V \rho \omega_{\mathbf{q}}}} a_{\mathbf{q} s}^{+} e^{-i \mathbf{q a}}\right) \Psi_{m}\left|n_{q}\right\rangle d \mathbf{r} .
\end{aligned}
$$

Здесь $\left|n_{q}\right\rangle$ - волновая функция фононов, $n_{q}$ - число фононов в моде с волновым вектором q (при отсутствии взаимодействия между электронами и фононами волновая функция представляет собой произведение электронной и фононной волновых функций). Так как волновая функция состояния мелкого донора имеет вид (1), выражение (6) можно переписать в следующем виде:

$$
\begin{aligned}
M_{n m}= & \sum_{i j} \alpha_{i} \beta_{j} \int\left\langle n_{q}+1\right| F_{n}^{i}(\mathbf{r})^{*} \varphi_{i}^{*}(\mathbf{k}, \mathbf{r}) \\
& \times\left(\sum_{\mathbf{a} \alpha} \mathbf{F}\left(\mathbf{r}-\mathbf{R}_{\mathbf{a} \alpha}\right) \mathbf{e}_{s \mathbf{q}} \sqrt{\frac{\hbar}{2 N V \rho \omega_{\mathbf{q}}}} a_{\mathbf{q} s}^{+} e^{-i \mathbf{q a}}\right) \\
& \times \mathbf{F}_{m}^{j}(\mathbf{r}) \varphi_{j}(\mathbf{k}, \mathbf{r})\left|n_{q}\right\rangle d \mathbf{r}
\end{aligned}
$$

Здесь $F_{n}^{i}(\mathbf{r})$ и $F_{m}^{j}(\mathbf{r})$ - огибающие волновых функций состояний доноров для уровней $n$ и $m$, принадлежащих долинам $i$ и $j$ соответственно, $\alpha_{i}$ и $\beta_{j}-$ коэффициенты, отражающие распределение волновой функции по долинам в $k$-пространстве и определяемые формулами в табл. 1. Поменяем в последнем выражении интегрирование по $\mathbf{r}$ и суммирование по а, также учтем тот факт, что огибающие волновых функций плавные (почти не изменяются на масштабах элементарной ячейки кристалла), a функция $\mathbf{F}\left(\mathbf{r}-\mathbf{R}_{\mathbf{a} \alpha}\right)$, напротив, очень „быстрая“ и существенно отличается от нуля только в окрестности точки $\mathbf{r}-\mathbf{R}_{\mathbf{a} \alpha}=0$. Это дает возможность вынести огибающие за знак интеграла, переписав выражение (7) в виде

$$
\begin{aligned}
M_{n m} & =\sum_{i j} \alpha_{i} \beta_{j} M_{i j} \\
& =\sum_{i j} \alpha_{i} \beta_{j} \sum_{\mathbf{a}} F_{n}^{i}(\mathbf{r})^{*} F_{m}^{j}(\mathbf{r}) e^{-i \mathbf{q a}} \int\left\langle n_{q}+1\right| \varphi_{i}^{*}(\mathbf{k}, \mathbf{r}) \\
& \times\left(\sum_{\alpha} \mathbf{F}\left(\mathbf{r}-\mathbf{R}_{\mathbf{a} \alpha}\right) \mathbf{e}_{s \mathbf{q}} \sqrt{\frac{\hbar}{2 N V \rho \omega_{\mathbf{q}}}} a_{\mathbf{q} s}^{+}\right) \varphi_{j}(\mathbf{k}, \mathbf{r})\left|n_{q}\right\rangle d \mathbf{r},
\end{aligned}
$$

где введено обозначение $M_{i j}$ - матричный элемент перехода между состояниями, описываемыми волновыми функциями, которые образованы вкладами лишь $i$-й и $j$-й долин. Далее для взаимодействия с длинноволновыми фононами (внутридолинные переходы) в кристаллах, в которых экстремумы зоны проводимости лежат на оси симметрии (например, кремний и германий), интеграл в последней формуле (8) следующим образом выражается через введенные Херингом [16] продольный, $\Xi_{u}$, и сдвиговый, $\Xi_{d}$, деформационные потенциалы [17]:

для взаимодействия с продольными фононами

$$
\begin{aligned}
\int\left\langle n_{q}\right. & +1 \mid \varphi_{i}^{*}(\mathbf{k}, \mathbf{r})\left(\sum_{\alpha} \mathbf{F}\left(\mathbf{r}-\mathbf{R}_{\mathbf{a} \alpha}\right) \mathbf{e}_{s \mathbf{q}} \sqrt{\frac{\hbar}{2 N V \rho \omega_{\mathbf{q}}}} a_{\mathbf{q} s}^{+}\right) \\
& \times \varphi_{j}(\mathbf{k}, \mathbf{r})\left|n_{q}\right\rangle d \mathbf{r}=i q \sqrt{\frac{\hbar\left(n_{q}+1\right)}{2 N V \rho \omega_{\mathbf{q}}}}\left(\Xi_{u} \cos ^{2} \vartheta+\Xi_{d}\right)
\end{aligned}
$$


для взаимодействия с поперечными фононами:

$$
\begin{aligned}
\int\left\langle n_{q}\right. & +1 \mid \varphi_{i}^{*}(\mathbf{k}, \mathbf{r})\left(\sum_{\alpha} \mathbf{F}\left(\mathbf{r}-\mathbf{R}_{\mathbf{a} \alpha}\right) \mathbf{e}_{s \mathbf{q}} \sqrt{\frac{\hbar}{2 N V \rho \omega_{\mathbf{q}}}} a_{\mathbf{q} s}^{+}\right) \\
& \times \varphi_{j}(\mathbf{k}, \mathbf{r})\left|n_{q}\right\rangle d \mathbf{r}=i q \sqrt{\frac{\hbar\left(n_{q}+1\right)}{2 N V \rho \omega_{\mathbf{q}}}}\left(\frac{1}{2} \Xi_{u} \sin 2 \vartheta\right) .
\end{aligned}
$$

Здесь $\vartheta-$ угол между волновым вектором $\mathbf{q}$ и осью симметрии долины; $\Xi_{u}$ и $\Xi_{d}$ имеют следующие значения: $\Xi_{u}=16.5$ эB, $\Xi_{d}=-4.3$ эВ [18].

Вероятность перехода между состояниями находится посредством золотого правила Ферми:

$$
\begin{aligned}
P_{m n} & =\frac{2 \pi}{\hbar} \sum_{\mathbf{q}}\left|M_{n m}\right|^{2} \delta\left(E-\hbar \omega_{\mathbf{q}}\right) \\
& =\frac{V}{(2 \pi)^{2} \hbar} \int\left|M_{n m}\right|^{2} \delta\left(E-\hbar \omega_{\mathbf{q}}\right) d^{3} q \\
& =\frac{V}{(2 \pi)^{2} \hbar} \int\left|\sum_{i j} \alpha_{i} \beta_{j} M_{i j}\right|^{2} \delta\left(E-\hbar \omega_{\mathbf{q}}\right) d^{3} q .
\end{aligned}
$$

Здесь приведено суммирование по всем конечным состояниям (всем волновым векторам излучаемого фонона), $E$ - энергия перехода. Для внутридолинных переходов $M_{i j}=0$ при $i \neq j$, следовательно, последнюю формулу для внутридолинных переходов можно переписать в виде

$$
\begin{aligned}
P_{m n} & =\frac{V}{(2 \pi)^{2} \hbar} \int\left|\sum_{i} \alpha_{i} \beta_{i} M_{i i}\right|^{2} \delta\left(E-\hbar \omega_{\mathbf{q}}\right) d^{3} q \\
& =\frac{V}{(2 \pi)^{2} \hbar} \int \sum_{i j} \alpha_{i} \beta_{i} \alpha_{j} \beta_{j} M_{i i} M_{j j}^{*} \delta\left(E-\hbar \omega_{\mathbf{q}}\right) d^{3} q .
\end{aligned}
$$

Как видно из последней формулы, полная вероятность перехода определяется не только квадратами матричных элементов переходов между однодолинными состояниями внутри одной долины, но и произведениями матричных элементов переходов между однодолинными состояниями в различных долинах (здесь однодолинными состояниями названа часть волновой функции примесного состояния, образованная вкладом лишь одной долины, т.е. одно слагаемое выражения (1)). Это обстоятельство приводит к вычислительным сложностям при расчете вероятностей внутридолинных переходов при взаимодействии с акустическими фононами, так как фонон с определенным волновым вектором, приводящий к переходу внутри одной долины, приводит также к переходу в другой. При этом матричные элементы переходов в различных долинах с излучением одного и того же фонона не равны, вследствие чего зависимость полного матричного элемента перехода от ориентации волнового вектора в пространстве фонона становится очень нетривиальной, сильно зависящей от направления вектора q и меняющейся при этом на несколько порядков. Однако из-за того, что долины зоны проводимости германия обладают сильной анизотропией, и того, что продольный и сдвиговый деформационные потенциалы различаются почти в 4 раза, значения матричных элементов переходов внутри одной долины становятся существенными в относительно небольшой области углов, задающих ориентации волнового вектора фонона, а именно, когда ориентации волновых векторов близки к оси симметрии долины. Это значит, что если при определенном направлении волнового вектора фонона матричный элемент в одной долине дает существенную величину, то матричный элемент в другой долине при том же волновом векторе будет, как правило, гораздо меньше. Как следствие, величины произведений $M_{i i} M_{j j}^{*}$ при $i \neq j$ становятся достаточно малыми по сравнению c $\left|M_{i i}\right|^{2}$ и в большинстве случаев первыми можно пренебречь. Выражение (11) приближенно можно представить в виде

$$
P_{m n} \approx \frac{V}{(2 \pi)^{2} \hbar} \int \sum_{i}\left|\alpha_{i} \beta_{i} M_{i i}\right|^{2} \delta\left(E-\hbar \omega_{\mathbf{q}}\right) d^{3} q .
$$

Формула (12) справедлива для переходов между состояниями, ось симметрии которых совпадает с осью симметрии долины, т.е. в которых квантовое число $\mathrm{m}$ равняется нулю. Если $m \neq 0$, при расчете темпов внутридолинных переходов по формуле (12) нужно проявлять особую аккуратность и в каждом конкретном случае проверять применимость этой формулы. В противном случае необходимо пользоваться более общим выражением (11).

\section{3. Результаты и заключение}

Далее на рис. 3-5 представлены рассчитанные темпы внутрицентровых переходов в германии, легированном мышьяком, при излучении длинноволновых акустических фононов в зависимости от междолинного расщепления, вызванного одноосной деформацией сжатия в кристаллографическом направлении [111]. Давление в этом направлении в 1 кбар соответствует приблизительно междолинному расщеплению 10.81 мэВ. Точки показывают расчетные значения, линии же представляют собой базисные сплайны, построенные по этим точкам. Формы рассчитанных кривых определяются зависимостью от деформации энергий переходов между состояниями донорного центра и коэффициентов $\alpha_{i}(i$ принимает значения от 1 до 4), определяющих вклад долин зоны проводимости германия в волновую функцию состояния донора. Темп релаксации состояния $2 p_{ \pm}\left(\Gamma_{3}\right)$ определяется переходами в состояния $3 p_{0}\left(\Gamma_{3}\right)$ (темп релаксации $\left.6 \cdot 10^{8} \mathrm{c}^{-1}\right), 2 p_{0}\left(\Gamma_{3}\right)\left(3.2 \cdot 10^{7} \mathrm{c}^{-1}\right), 2 s\left(2.6 \cdot 10^{8} \mathrm{c}^{-1}\right), 3 s$ $\left(1.2 \cdot 10^{7} \mathrm{c}^{-1}\right)$. Зависимость темпов релаксации от деформации в рамках используемой модели очень слабая, поэтому ею пренебрегается. 


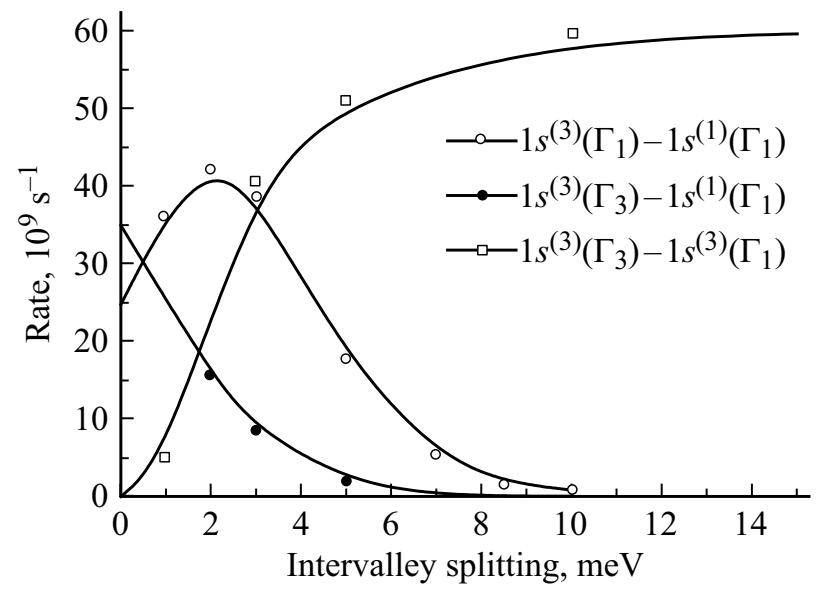

Рис. 3. Зависимости от междолинного расщепления темпов релаксации состояний триплетной группы $1 s$.

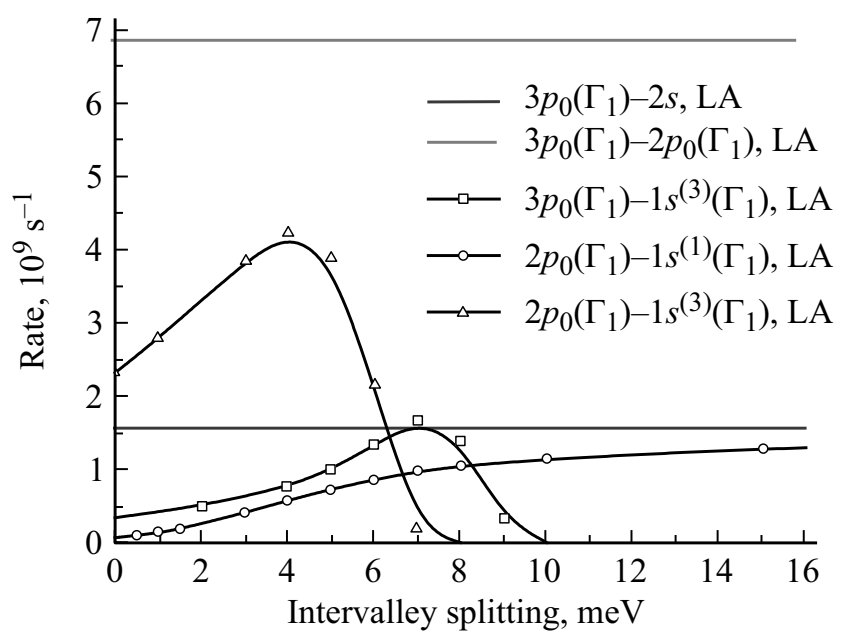

Рис. 4. Зависимости от междолинного расщепления темпов релаксации состояний $2 p_{0}$ и $3 p_{0}$ донора мышьяка, связанных с нижней долиной зоны проводимости германия.

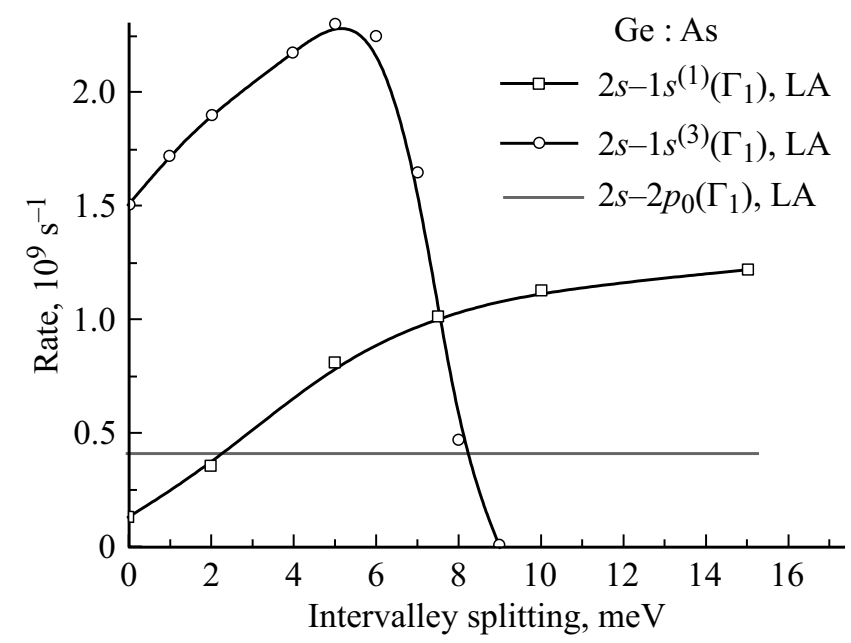

Рис. 5. Зависимость от междолинного расщепления темпов релаксации состояния $2 s$ донора мышьяка, связанного с нижней долиной зоны проводимости германия.
На основе вычисленных темпов релаксации в рамках уравнений баланса проведен расчет населенностей состояний донора. Показано, что при фотоионизации доноров мышьяка формируется инверсная населенность состояний примеси. Однако при одноосной деформации вдоль кристаллографического направления [111] между состояниями $2 p$, связанными с нижней долиной зоны проводимости германия (долиной, вдоль которой приложено давление), и основным состоянием нет промежуточных уровней (см. рис. 2), что исключает возможность реализации четырехуровневой лазерной схемы, в отличие от случая недеформированного кристалла. Поэтому с точки зрения обнаружения лазерного эффекта в таком материале недеформированный германий видится более перспективным. Возможно, что четырехуровневая схема также может быть реализована в деформированном германии в направлении $\{110\}$, но в данной работе такое направление деформации не анализируется.

В систему балансных уравнений были включены уровни донора начиная от основного состояния $1 s(A)$ и заканчивая состоянием $2 p_{ \pm}$. Состояния, лежащие выше уровня $2 p_{ \pm}$, и континуум состояний заменялись дополнительным эффективным уровнем. Всего система балансных уравнений включала 12 уровней и учитывала вырождение состояний донора. Даже при нулевой деформации кристалла четырехкратно вырожденные состояния разделялись на два уровня, один из которых подобно случаю одноосно деформированного кристалла в направлении [111] связан с синглетным состоянием, образованным вкладом лишь одной долины, другой с состоянием триплета, связанным с оставшимися тремя долинами. Подобное разделение, согласно формулам в табл. 1, может быть выбрано и при нулевой деформации. Конечно, при нулевой деформации можно было бы выбрать и другие комбинации долинных вкладов в состояния доноров, т.е. другие значения $\alpha_{i}$, но для поиска инверсии населенностей и связанного с ней эффекта стимулированного излучения такой вариант удобен. Удобство обусловлено тем, что подобно одноосной деформации поляризация излучаемого на внутрицентровых переходах фотона также выделяет в системе ось. Из-за этого матричные элементы излучательного перехода между состояниями, связанными с разными долинами, различны. При переходе между состояниями с нулевым значением квантового число $m$ излучение с большей вероятностью происходит на поляризации, ориентированной вдоль оси той долины, с которой связаны состояния перехода, а если $m \neq 0$, то на поляризации, ориентированной ортогонально. Поэтому удобно, чтобы состояния, на переходах между которыми ожидается усиление, были образованы вкладом лишь этой одной долины. Состояния же, которые связаны с оставшимися тремя долинами, образуют параллельный релаксационный канал, на котором, как полагается в используемой модели, излучения фотонов не происходит.

На рис. 6 показана зависимость инверсии населенностей между одним из уровней $2 p$-состояний доноров мы- 


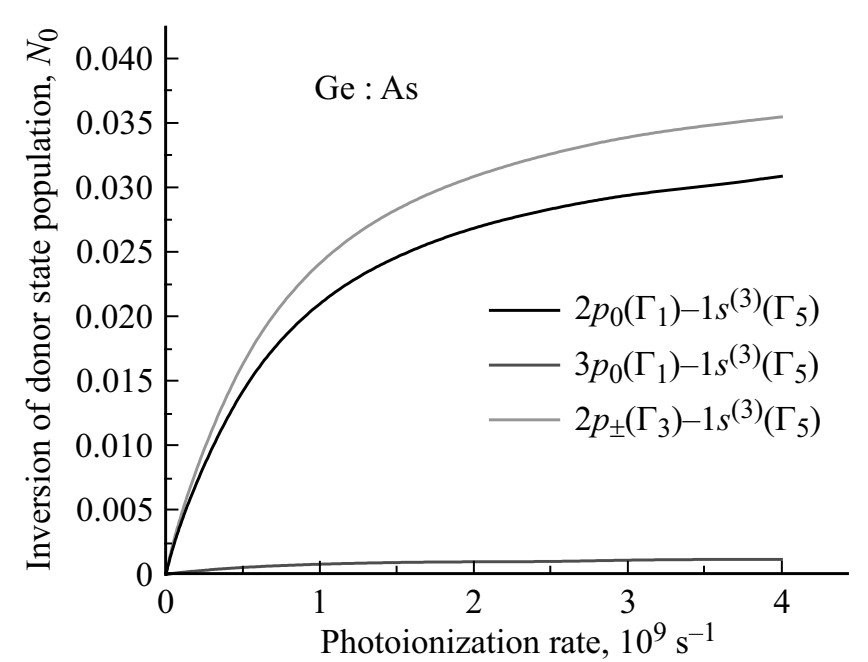

Рис. 6. Зависимости от темпа фотоионизации инверсии населенностей на различных оптически разрешенных переходах в донорах мышьяка в германии. Инверсия выражена в единицах концентрации легирования $\left(N_{0}\right)$.

шьяка в германии и уровнем, связанным с триплетным состоянием $1 s\left(T_{2}\right)$, от темпа фотоионизации доноров.

Результат расчета населенностей уровней при оптическом возбуждении зависит от специфики захвата фотоионизованных носителей и релаксации высоковозбужденных состояний доноров. Переходы между уровнями доноров при излучении фононов тем эффективнее, чем более схожа топология волновых функций состояний, участвующих в переходе. Это значит, что, например, при прочих равных условиях из состояния $p$-типа с большей вероятностью будут происходить переходы в состояния тоже $p$-типа, из состояний $s$-типа - с большей вероятностью в $s$-состояния, из состояний с определенным значением квантового числа $m$ с большей вероятностью переходы будут происходить в состояния с таким же $m$ и т.д. Это приводит к тому, что релаксация высоковозбужденных состояний происходит не каскадным образом, в процессе релаксации возникают различные потоки носителей по различным наборам энергетических уровней - релаксационные каналы. Возникает вопрос - какая часть носителей в процессе релаксации проходит через каждое состояние. В данной работе предполагалось, что в процессе захвата с равной вероятностью заселяются состояния всех типов, что приводит к равной вероятности всех релаксационных каналов. В использованной модели это свойство свелось к тому, что вероятности перехода с самого верхнего эффективного уровня в балансной схеме, связанного с континуумом и верхними возбужденными состояниями (лежащие выше уровня $2 p_{ \pm}$), в состояния типов $p_{0}$, $s$ и $p_{ \pm}$с точностью до кратности вырождения уровня считались равными.

Как видно из результатов расчетов, максимальная инверсия населенностей достигается на пере- ходе $2 p_{ \pm}\left(\Gamma_{3}\right)-1 s^{(3)}\left(\Gamma_{5}\right)$, хотя значения инверсии на этом переходе близки к значениям на переходе $2 p_{0}\left(\Gamma_{1}\right)-1 s^{(3)}\left(\Gamma_{5}\right)$. Из-за описанных выше сложностей корректного учета особенностей захвата и релаксации высоковозбужденных состояний превалирование перехода $2 p_{ \pm}\left(\Gamma_{3}\right)-1 s^{(3)}\left(\Gamma_{5}\right)$ требует экспериментальной проверки.

Работа выполнена при поддержке Российского фонда фундаментальных исследований (№ 18-42-520064).

\section{Список литературы}

[1] A.P. Heberle, J.J. Baumberg, E. Binder, T. Kuhn, K. Kohler, K.H. Ploog. IEEE J. Select. Top. Quant. Electron., 2, 769 (1996).

[2] A.M. Stoneham, A.J. Fisher, P.T. Greenland. J. Phys. Condens. Matter, 15, L447 (2003).

[3] L.C.L. Hollenberg, C.J. Wellard, C.I. Pakes, A.G. Fowler. Phys. Rev. B, 69, 233301 (2004).

[4] J.J. Pla, K.Y. Tan, J.P. Dehollain, W.H. Lim, J.J.L. Morton, F.A. Zwanenburg, D.N. Jamieson, A.S. Dzurak, A. Morello. Nature, 496, 334 (2013).

[5] A.J. Sigillito, A.M. Tyryshkin, J.W. Beeman, E.E. Haller, K.M. Itoh, S.A. Lyon. Phys. Rev. B, 94, 125204 (2016).

[6] K.J. Morse et al. Sci. Adv. Quant. Phys., 3, e1700930 (2017).

[7] S.G. Pavlov, H.-W. Hubers, M.H. Rummeli, J.N. Hovenier, T.O. Klaassen, R.Kh. Zhukavin, A.V. Muravjov, V.N. Shastin. In: Towards the First Silicon Laser, ed. by L. Pavesi, S. Gaponenko, L. Dal Negro. [NATO Sci. Ser. II: Mathematics, Physic and Chemistry (Kluwer Academic Publishers), 93 (2003) p. 331.

[8] S.G. Pavlov, N. Deßmann, V.N. Shastin, R.Kh. Zhukavin, B. Redlich, A.F.G. van der Meer, M. Mittendorff, S. Winnerl, N.V. Abrosimov, H. Riemann, H.-W. Hübers. Phys. Rev. X, 4, 021009 (2014).

[9] K.A. Kovalevsky, R.Kh. Zhukavin, V.V. Tsyplenkov, V.N. Shastin, N.V. Abrosimov, H. Riemann, S.G. Pavlov, H.-W. Hubers. Semiconductors, 47 (2), 235 (2013).

[10] M. Fuechsle, J.A. Miwa, S. Mahapatra, H. Ryu, S. Lee, O. Warschkow, L.C.L. Hollenberg, G. Klimeck, M.Y. Simmons. Nat. Nanotechnol., 7, 242 (2012).

[11] Р.Х. Жукавин, К.А. Ковалевский, С.М. Сергеев, Ю.Ю. Чопорова, В.В. Герасимов, В.В. Цыпленков, Б.А. Князев, Н.В. Абросимов, С.Г. Павлов, В.Н. Шастин, Г. Шнайдер, Н. Дессманн, О.А. Шевченко, Н.А. Винокуров, Г.Н. Кулипанов, Г.-В. Хьюберс. Письма ЖЭТФ, 106, 555 (2017).

[12] M. Gienger, P. Gross, K. Lassmann. Phys. Rev. Lett., 64, 1138 (1990).

[13] R. Barrie, R. Nishikawa. Canadian J. Phys., 41, 1135 (1963).

[14] R.J. Bell, W.T. Bousman, jr., G.M. Goldman, D.G. Rathbun. Surf. Sci., 7, 293 (1967).

[15] Б.И. Шкловский, А.Л. Эфрос. Электронные свойства легированных полупроводников (М., Наука, 1979) § 4, c. 38 .

[16] C. Herring, E. Vogt. Phys. Rev., 101, 944 (1956).

[17] В.Н. Абакумов, В.И. Перель, И.Н. Ясиевич. Безызлучательная рекомбинация в полупроводниках (Петербургский ин-т ядерной физики Б.П. Константинова РАН, СПб., 1997) Приложение 3, с. 329. 
[18] Y.S. Choi, J.-S. Lim, T. Numata, T. Nishida, S.E. Thompson. J. Appl. Phys., 102, 104507 (2007).

Редактор Л.В. Шаронова

\section{Intracenter relaxation of shallow arsenic donors in stressed germanium. Inversion of population under optical excitation}

V.V. Tsyplenkov, V.N. Shastin

Institute for Physics of Microstructures,

Russian Academy of Sciences,

603950 Nizhny Novgorod, Russia

Abstract Long-wave acoustic phonons assisted relaxation rates of the lower excited states $1 s(T), 2 p_{0}, 2 s, 3 p_{0}, 2 p_{ \pm}$of arsenic donors in a germanium crystal are calculated as functions of an uniaxial stress in the crystallographic direction [111]. Estimates of the donor states populations under optical excitation were made on the base of the calculated times. It is shown theoretically that optical excitation leads to the inversion of arsenic donor states population, and at zero stress it is possible to realize a four-level laser scheme with a radiative transition between $2 p$ states and $1 s$ triplet state. Under optical excitation by $\mathrm{CO}_{2}$ laser radiation the value of the expected gain estimated as $\sim 0.35 \mathrm{~cm}^{-1}$ at the donor concentration of $2 \cdot 10^{15} \mathrm{~cm}^{-3}$, at the radiation frequency $1.98 \mathrm{THz}$ in the case of $2 p \pm 1 s(T)$ laser transition and at the radiation frequency $1.25 \mathrm{THz}$ in the case of $2 p_{0} \rightarrow 1 s(T)$ laser transition. 